\title{
Terapia de Interacción Guiada en díadas madre-bebé con sintomatología depresiva materna en el embarazo: un estudio piloto ${ }^{1}$
}

\section{Guided Interaction Therapy in mother-infant dyads with maternal depressive symptoms in pregnancy: a pilot study}

\author{
Marcia Olhaberry Huber ${ }^{2}$ \\ Constanza Mena \\ Johana Zapata \\ Ángela Miranda \\ Macarena Romero \\ Pontificia Universidad Católica de Chile, Santiago, Chile \\ Catalina Sieverson \\ Universidad de Chile, Santiago, Chile
}

(Rec: abril de 2015 - Acept: agosto de 2015)

\begin{abstract}
Resumen
La depresión perinatal constituye un trastorno del ánimo de alta frecuencia, que afecta negativamente a la madre y al bebé, y requiere una intervención dirigida a ambos miembros de esta díada. Esta investigación evalúa la aplicabilidad y resultados de una intervención piloto dirigida a fortalecer el vínculo y a reducir la depresión en díadas con esta problemática. Se estudian tres díadas madre-hijo/a, con sintomatología depresiva materna y se desarrolla una intervención psicoterapéutica breve utilizando el modelo de Interacciones Guiadas. Los resultados muestran una reducción en la sintomatología depresiva y un aumento en sensibilidad materna, cooperatividad infantil y calidad vincular. La intervención realizada resulta eficaz en las variables estudiadas, requiriéndose nuevas aplicaciones en muestras de mayor tamaño para confirmar estos hallazgos.
\end{abstract}

Palabras clave: depresión perinatal, sensibilidad materna, interacción guiada.

\begin{abstract}
Perinatal depression is a high frequency mood disorder which negatively affects mother and baby, and requires an intervention addressing both members of the dyad. This paper evaluates the applicability and results of a pilot intervention to strengthen this bond and reduce depression in dyads with this problem. Three motherchild dyads, with maternal depressive symptoms and a brief psychotherapeutic intervention using the model of Guided Interactions were studied. The results showed a reduction in depressive symptoms and an increase in maternal sensitivity, child cooperativeness and relationship quality. The intervention turned out to be effective in the variables under study. Nevertheless, new applications over larger sample sizes are required to confirm these findings.
\end{abstract}

Keywords: perinatal depression, maternal sensitivity, guided interactions.

\footnotetext{
1 Este trabajo forma parte de los proyectos de investigación FONDECYT N ${ }^{\circ} 11140230$ y N³120109, financiados por CONICYT y por la Iniciativa Científica Milenio, proyecto IS 130005, financiado por el Fondo de Innovación para la Competitividad (FIC) del Ministerio de Economía, Fomento y Turismo.

2 Correspondencia a: Marcia Olhaberry Huber. Av. Vicuña Mackenna 4860, Macul, Santiago, Chile. Email: mpolhabe@uc.cl
} 


\section{Antecedentes}

La depresión perinatal constituye un trastorno del ánimo que se presenta durante el embarazo y/o con posterioridad al parto. No está definida como tal en los manuales diagnósticos, sin embargo, comparte las características de la depresión unipolar. Adicionalmente, los síntomas descritos en la depresión perinatal pueden ser considerados como parte de las vivencias normales durante el embarazo o el puerperio, dificultando así su pesquisa y diagnóstico (Halbreich \& Karkun, 2006).

Estudios internacionales muestran que entre un $6 \%$ y un $38 \%$ de las mujeres sufren de depresión, durante o después del embarazo, duplicándose estas cifras en los países en vías de desarrollo (Evans, Vicuña \& Marín, 2003; Field, 2011; Rojas et al., 2007). Las investigaciones muestran además, que quienes presentan un cuadro depresivo durante el embarazo tienen una mayor probabilidad de mantenerlo después del parto, señalando estudios chilenos que un $40 \%$ de las mujeres presenta síntomas de depresión o ansiedad durante el puerperio (Alvarado, Rojas, Monardes, Perucca, Neves, Olea \& Vera, 2000; Minsal, 2013).

La depresión perinatal, impacta también al bebé, observándose en los hijos/as de madres deprimidas un mayor riesgo de presentar bajo peso al nacer, rezagos en el desarrollo y problemas de salud mental (Earls, 2010; Field, Diego \& Hernández-Reif, 2006). El vínculo madre-bebé también se ve afectado, reportando las investigaciones competencias maternas insuficientes y baja calidad de los cuidados (Minkovitz et al., 2006), mayor hostilidad en la crianza (Cornishet al., 2006), baja sensibilidad (Pearson, Melotti, Heron, Joinson, Stein, Ramchandani \& Evans, 2012), mayor riesgo de desarrollar un estilo de apego inseguro (Muzik \& Borovska, 2010; Santelices, Olhaberry, Araneda, Tapia \& Pérez-Salas, 2007), junto con un mayor riesgo de presentar psicopatologías (Biedermann, Martínez, Olhaberry \& Cruzat, 2009).

A su vez, la adecuada sensibilidad materna implica la lectura e interpretación de las señales, la capacidad de responder contingentemente y de manera ajustada a las necesidades infantiles, generando círculos virtuosos de interacción que favorecen sintonía y el placer compartido, impactando positivamente la calidad vincular (Cohn, Matias, Tronick, Connell \& Lyons- Ruth, 1986; Di Carlo Onwujuba \& Baumgartner, 2013).

Buscando profundizar en la construcción de los vínculos tempranos, autores contemporáneos han desarrollado el concepto de mentalización o función reflexiva para explicar la capacidad de comprender el propio comportamiento y el de otros, en términos de estados mentales (Allen, Fonagy \& Bateman, 2008). En el caso de las madres, implica concebir al infante con deseos, intenciones y emociones más allá de sus necesidades, tomando distintas perspectivas de lo que ocurre en el mundo interno propio y del niño, así como en la realidad externa. Las interacciones que incluyen una adecuada función mentalizante en el adulto favorecen la respuesta sensible frente a las necesidades infantiles y también la construcción de un vínculo de apego seguro. Para que esto ocurra, el adulto debe ser capaz de reflexionar durante el encuentro con el niño/a acerca de los estados mentales en ambos, lo que muchas veces no ocurre en madres con sintomatología depresiva (Allen \& Fonagy, 2002; Fonagy, Bateman \& Bateman, 2011).

En cuanto a las intervenciones desarrolladas para tratar depresión perinatal, éstas se han centrado mayoritariamente en la madre y en menor medida en la relación madre-bebé, recuperando en algunos casos el estado de ánimo materno, pero no siempre favoreciendo la salud mental infantil y la calidad vincular (Olhaberry et al., 2013).

Las intervenciones dirigidas a promover vínculos tempranos saludables, destacan la importancia de considerar la sensibilidad y la capacidad de mentalización materna para lograr resultados favorables (Fonagy, 1997; O’Connor \& Zeanah, 2003). En esta línea, la utilización de retroalimentación a través de videos o videofeedback ha demostrado ser una herramienta terapéutica útil para promover estas habilidades (Juffer, Bakermans-Kranenburg \& van IJzendoorn, 2008; McDonough, 2004).

En el caso específico de la depresión perinatal, la evidencia indica que luego de una intervención en $v i$ deofeedback en la modalidad de Interacciones Guiadas (IG), las madres descubren una imagen más positiva de sí mismas y disfrutan más del tiempo que pasan con sus hijos, lo que impacta positivamente en el vínculo (Vik \& Braten, 2009; Vik \& Hafting, 2006). En Chile se ha aplicado esta técnica en familias vulnerables, mostrando ser efectiva en mejorar la sensibilidad materna (Suárez, Muñoz, Gómez \& Santelices, 2009), pero no contamos con aplicaciones específicas en díadas con depresión perinatal. Considerando estos antecedentes, se evalúa la aplicabilidad y resultados de una intervención piloto en IG en díadas madre-hijo/a con esta problemática. 


\section{Método}

\section{Participantes}

El presente estudio corresponde a la tercera intervención desarrollada en el contexto de un estudio longitudinal para la reducción de la depresión y la promoción de un vínculo seguro madre-bebé. Las participantes fueron invitadas al programa por presentar sintomatología depresiva durante el embarazo (BDI z5) y al menos un factor de riesgo detectado por la Escala de Evaluación de Riesgo Psicosocial EPSA, utilizada regularmente en los consultorios chilenos. La participación fue voluntaria y todas firmaron una carta de consentimiento informado antes de iniciar las mediciones y la intervención. El programa consideró como retribución a las participantes la entrega de dinero para los traslados y el acceso a redes de derivación expeditas para atenciones médicas y psicológicas.

Se estudian tres díadas madre-hijo/a, atendidas en Centros de Salud Pública de Santiago de Chile. Considerando la influencia de la presencia del padre en la calidad del vínculo madre-bebé (Olhaberry \& Santelices, 2013), se seleccionaron tres díadas cuyas madres se encontraban en una relación de pareja estable con el padre del niño/a. Sus edades variaron entre 23 y 24 años y las de los bebés entre los 7 y 12 meses.

\section{Instrumentos}

Las madres fueron evaluadas durante el embarazo y las díadas al mes de nacidos los bebés (después de la $1^{\mathrm{a}}$ intervención grupal), a los seis meses postparto (después de la $2^{\mathrm{a}}$ intervención grupal) y al finalizar la intervención en IG. Se utilizaron los siguientes instrumentos:

Ficha de Antecedentes: contiene información socio demográfica y antecedentes en salud.

Inventario para la Depresión de Beck, BDI-I (Beck, Ward, Mendelson, Mock \& Erbaugh, 1961): consta de 21 ítems y evalúa la sintomatología depresiva actual. El puntaje total varía entre 0 y 63 , y se definen cuatro categorías: mínima o no deprimida $0-9$, leve 10-18, moderada 19-29 y severa 30-63. Los análisis de fiabilidad son adecuados, reportando un valor $\alpha=.90$.

Cuestionario OQ-45.2, (Lambert, Hansen, Umpress, Lunnen, \& Okiishi, 1996): validado en Chile por Von Bergen y De la Parra (2002). Consta de 45 ítems y evalúa tres áreas de funcionamiento: sintomatología ansiosa y depresiva, relaciones interpersonales y ajuste al rol social. Arroja puntajes entre 0 a 180 puntos y define como punto de corte 73 puntos. Posee características psicométricas adecuadas $(\alpha=.90)$.

CARE-Index Infantes, desde el nacimiento a los 15 meses (Crittenden, 2006): considera tres a cinco minutos de grabación en video de la interacción de juego entre el adulto y el niño. El sistema de codificación define tres descriptores para el adulto: sensible, controlador y no responsivo, y cuatro para el infante: cooperativo, difícil, compulsivo y pasivo. Considera una escala de sensibilidad diádica de 0 a 14 puntos, indicando 0-4 "riesgo", 5-6 "inepto", 7-10 "adecuado" y 11-14 "sensible", puntajes menores a 7 indican necesidad de intervención. Las codificaciones de los videos fueron realizadas por psicólogas capacitadas por la autora del instrumento, obteniendo una confiabilidad $\geq$ 0.7. La confiabilidad indica la correlación obtenida por el codificador en relación a un gold standard definido por la autora.

Escala de experiencias en las relaciones cercanas, forma abreviada, ECR-S (Wei, Russell, Mallinckrodt, \& Vogel, 2007): evalúa el patrón de apego adulto considerando dos dimensiones: Ansiedad y Evitación, asociadas a patrones de apego inseguros. Puntajes altos en ambas o en una de estas dimensiones indican apego inseguro y puntajes bajos, apego seguro. Los coeficientes alpha para la sub-escala Ansiedad varían desde .77 a .86 , y para la sub-escala Evitación entre .78 y .88 en la versión utilizada.

\section{Procedimiento}

Se consideró la intervención en IG desarrollada por McDonough $(1993,2004)$ y el Modelo ODISEA desarrollado en Chile por Gómez y Muñoz (2013), realizando una adaptación de cuatro sesiones. Fue implementada en los domicilios por una dupla de psicólogas quienes participaron en reuniones clínicas semanales para el análisis y discusión de los casos.

Primera Sesión. Se exploran las preocupaciones de la madre sobre el niño/a o sobre la relación, luego se graba un video de interacción de juego madre-hijo/a con un set de juguetes preparado para la edad y finalmente se comenta la experiencia y se define una tarea.

Trabajo post sesión: Las terapeutas identifican las secuencias negativas y positivas en el video, buscando ligar las interacciones observadas a la preocupación de la madre. Se consideran secuencias negativas aquellas en que se observa algunos de los siguientes aspectos: baja sincronía, afecto negativo, comportamiento hostil o controlador en el adulto, evitación de miradas, 
ausencia de secuencias de turnos, dificultad para atraer la atención del niño, juego por sobre o por debajo de las habilidades del niño, incongruencia entre el lenguaje verbal y no verbal. Entre las secuencias positivas en la interacción se consideró: placer compartido, miradas compartidas con afecto positivo, atención conjunta, sincronía, intervenciones del adulto que contribuyen a la regulación infantil y al alivio del malestar, secuencias de turnos, vocalizaciones positivas, turnos vocales, uso de mentalizaciones adecuadas que enriquecen el intercambio, congruencia verbal y no verbal en el adulto, conductas reparadoras frente a momentos de tensión o desencuentro. Luego seleccionan segmentos de interacciones positivas, definiendo su uso terapéutico.

Sesión de retroalimentación: Se muestran las secuencias seleccionadas y se favorece la reflexión de la madre frente a los distintos segmentos, luego se define una nueva tarea.

En las sesiones tres y cuatro se repite la estructura de las dos primeras y se agrega un video de alimentación. La sesión cuatro también considera una evaluación del proceso y un cierre.

\section{Análisis de datos}

Se realizan análisis estadísticos descriptivos en relación a las variables estudiadas. Dado el pequeño tamaño muestral, se realizan análisis con pruebas no paramétricas (prueba de Friedman y Wilcoxon) para determinar la significación del cambio en las variables de interés luego de la intervención.

\section{Resultados}

Se presentan los resultados cualitativos de la intervención en IG y los resultados cuantitativos obtenidos en cada díada, considerando la evolución de la sintomatología depresiva materna y el vínculo durante el programa. Los antecedentes de las participantes al momento de la intervención en IG, se presentan en la tabla 1.

Descripción, evolución y resultados Díada 1: Alejandra y Agustina

Representaciones y calidad de la interacción: Las representaciones iniciales de Alejandra sobre su hija y su desempeño materno son predominantemente negativas, señalando: "se aburre", "me manipula" y "con todos se porta mejor que conmigo". Le cuesta lidiar con las necesidades de dependencia y se muestra exigente: "me gustaría que jugara un par de horas sola", destacando que en su infancia ella fue siempre muy independiente. La historia vincular de Alejandra interfiere en la interacción con Agustina, lo que se observa en la sobre exigencia de autonomía, en la baja sensibilidad acompañada de conductas hostiles, así como en la inhibición y algunas conductas difíciles de la niña observadas en la evaluación dos y tres, previas a la intervención en IG. De acuerdo a los puntajes obtenidos en las evaluaciones previas, la madre se ubica en la categoría "inepta" en la escala de sensibilidad diádica.

Tabla 1

Antecedentes socio demográficos y familiares.

\begin{tabular}{|c|c|c|c|}
\hline & $\begin{array}{c}\text { Díada } 1 \\
\text { Alejandra - Agustina }\end{array}$ & $\begin{array}{c}\text { Díada } 2 \\
\text { Juanita - Jimena }\end{array}$ & $\begin{array}{c}\text { Díada } 3 \\
\text { Sonia - Juan }\end{array}$ \\
\hline Edad M & 24 años & 23 años & 23 años \\
\hline Edad N & 7 meses & 9 meses & 12 meses \\
\hline Educación M & 13 años & 10 años & 12 años \\
\hline Ocupación M & $\begin{array}{l}\text { Dueña de casa/ } \\
\text { Estudiante }\end{array}$ & $\begin{array}{l}\text { Dueña de casa/ } \\
\text { Trabajo ocasional }\end{array}$ & $\begin{array}{l}\text { Dueña de casa/ } \\
\text { Trabajo ocasional }\end{array}$ \\
\hline$N^{o}$ de hijos & 1 & 2 & 2 \\
\hline Asiste a SC & Desde los $6 \mathrm{~m}$ & Desde los 7 meses & No \\
\hline Tipo de familia & Nuclear & Extensa & Nuclear \\
\hline Participación $\mathbf{P}$ & Juego/comida & Juego & Juego \\
\hline Patrón Apego M & Evitativo & Preocupado & Temeroso \\
\hline Rel. de pareja & Buena & Regular & Buena \\
\hline
\end{tabular}

“M”: Madre, "P”: Padre, “N”: Niño/a, "SC": Sala Cuna. 


\section{Resumen del proceso IG Díada 1}

Sesión 1: Alejandra señala que "nada resulta para calmar a Agustina" y que ella "está cansada". Relata que sólo ella tiene problemas para calmarla y no identifica estados internos en la niña ni necesidades más allá del aburrimiento. El juego transcurre en la cama matrimonial con una caja llena de juguetes, la madre se los muestra a la niña y los apila. Luego del juego, Alejandra dice que "generalmente Agustina juega sola". Cree que la niña "se sintió bien, estuvo tranquila y pocas veces la ve asî". Al final de la sesión señala que sacan los juguetes sólo el fin de semana, por lo que se le propone hacerlo también durante la semana.

Trabajo clínico post sesión 1: En las secuencias negativas Alejandra muestra a Agustina una gran cantidad de juguetes pero no propone un juego, la postura física dificulta el contacto visual y la niña no logra explorar el material. El tono emocional es plano en la niña, quien no vocaliza ni manifiesta iniciativa hacia algún juguete. La madre parece centrada en su propia experiencia, mueve los juguetes alrededor de Agustina y las actividades propuestas no son adecuadas para la edad de la niña. Las secuencias positivas consideran contacto visual, sonrisas compartidas, la entrega de juguetes, vocalizaciones que favorecen el disfrute y contenidos verbales de la madre ajustados a la experiencia de la niña.

Sesión 2 Retroalimentación, primer video: Asociado a la preocupación inicial de la madre, se identificaron los aspectos del comportamiento materno que promueven un estado emocional favorable en la niña, buscando fortalecer las representaciones positivas de ambas. Alejandra no logra ver su contribución al afecto positivo y placer en Agustina. Se le señala que ella explica la satisfacción de la niña en los juegos a partir de personas que no están presentes, se le pregunta: “¿con quién está la niña en las imágenes?”; responde: “conmigo", se emociona y dice: "me cuesta sentirme buena mamá". Logra también ligar los estados emocionales de ambas: "si estoy tensa, ella también, si me relajo, ella también se relaja".

Sesión 3: Se graba una interacción de alimentación en la que la madre da una sopa de verduras a la niña, quien la recibe con agrado. En un segundo momento la niña da mínimas señales de desinterés en la comida y la madre dice "no quieres más... ya quieres galleta" y entrega una galleta a la niña. Mientras la niña come la galleta, la interacción se empobrece y la niña está absorta y sin interés en la madre. Finalizada la comida, se filma una interacción de juego en que la madre le entrega otra galleta antes de comenzar. La madre refiere sentirse bien mientras le daba comida "hasta que Agustina se enojó y quería una galleta".

Trabajo clínico post sesión 3: La interacción es positiva durante la primera parte de la alimentación, la madre comenta con tono dulce lo que está ocurriendo, la niña sonríe y come disfrutando la sopa de verduras. Surgen algunos episodios de distracción que la madre logra manejar jugando y cantando. Las interacciones negativas se observan principalmente a partir de la incorporación de galletas, durante la comida y el juego. La madre busca jugar pero se frustra al no tener respuesta de la niña y verbaliza amenazas: "te voy a dejar sola".

Sesión 4 Retroalimentación, segundo video: Se muestran los momentos en que Alejandra habla de lo que le ocurre a Agustina y se trabaja sobre el valor de poner en palabras las experiencias emocionales de la niña y cómo esto resulta muy valioso para calmarla. En el video de interacción de juego se muestran segmentos en que la niña no tiene la galleta y logran interactuar positivamente. Se aborda la diferencia entre el juego con y sin galleta.

Cierre: Se trabajan con Alejandra los aspectos positivos en su interacción con Agustina destacando que busca opciones para atraer su atención, la estimula y pone en palabras lo que cree que le ocurre. Ella se muestra contenta y señala: "me di cuenta de las cosas buenas que hago con mi hija". La calidad vincular en esta díada mejora luego de la intervención con IG, evidenciando un beneficio a partir del trabajo terapéutico diádico y focalizado. Dichos cambios se reflejan en un aumento de la sensibilidad materna y cooperatividad infantil, desde la evaluación tres a la evaluación cuatro. Se observan aún dificultades para lidiar con los afectos displacenteros de la niña y transformarlos, recurriendo a la comida como un sustituto de la función que la madre no logra desempeñar. El detalle de los puntajes obtenidos por la Díada 1 en sensibilidad y cooperatividad en las evaluaciones dos, tres y cuatro se presenta en la figura 1. 


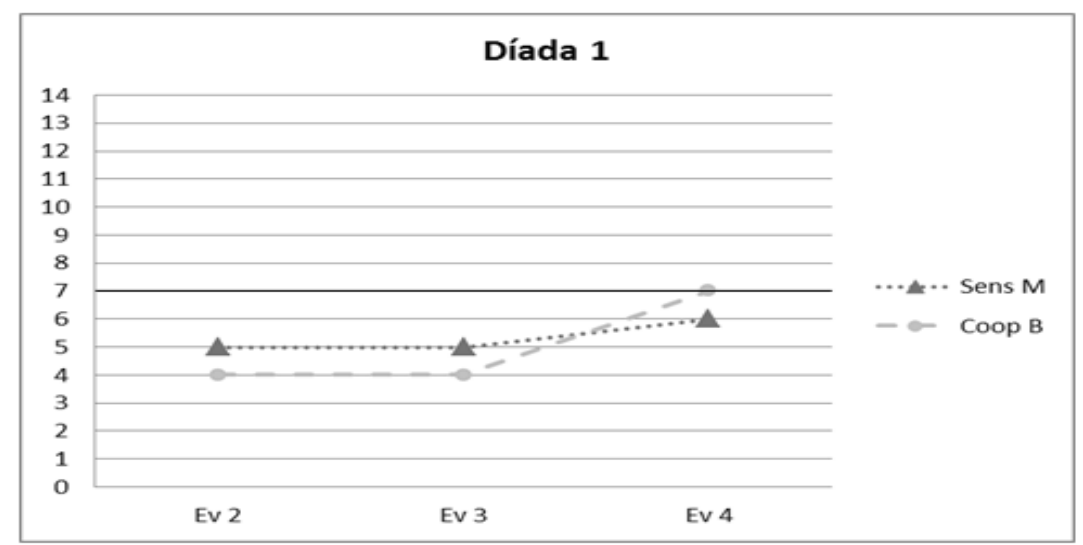

Figura 1. Puntajes obtenidos por la Díada 1 en sensibilidad materna y cooperatividad infantil en las evaluaciones 2, 3 y 4.

Descripción, evolución y resultados Díada 2: Juanita y Jimena

Representaciones y calidad de la interacción: Juanita describe a Jimena como una niña tranquila, sin embargo, le preocupan sus "deseos de hacer siempre lo que quiere" y su falta de herramientas para calmarla cuando llora. En las evaluaciones dos y tres, previas a la intervención en IG, el puntaje en sensibilidad de ubica en la categoría "inepta”. Se muestra poco expresiva, el juego es aburrido y por debajo de las capacidades de la niña, quien colabora poco.

\section{Resumen del proceso IG Díada 2}

Sesión 1: Juanita expresa preocupación por la necesidad de atención permanente de Jimena, sintiendo cansancio y un impacto negativo en su ánimo por estas exigencias. Durante la interacción de juego la madre explora los materiales en silencio, Jimena presta atención de manera intermitente y se trata de parar y caminar. La madre verbaliza "hacia allá no" y la retiene buscando nuevas propuestas de juego. Luego de la grabación, Juanita dice que su hija estuvo "muy inquieta" y "quería caminar y moverse hacia lugares peligrosos". Después de la conversación sobre el juego, Juanita dice que aprendió que su hija está "pasando a otra etapa" que quizás le llama más la atención conocer y explorar.

Trabajo clínico post sesión 1: En cuanto a las secuencias positivas, se eligen segmentos en que ambas se miran, vocalizan, comparten sonrisas y muestran un alto nivel de sincronía. En las secuencias negativas, la madre critica el alejamiento de Jimena e impide la exploración acercándola físicamente, la niña se queja, mira a la cámara o se queda inmóvil.

Sesión 2 Retroalimentación, primer video: Se trabaja con la madre en relación a la confianza en las habilidades de ambas y el desarrollo de estrategias para favorecer la autonomía, ligando la exploración en la niña con el manejo del malestar y el desarrollo de mecanismos auto regulatorios. Se muestran secuencias en que la niña quiere estar de pie, y la madre lo permite, expresando ambas alegría frente al logro. Se reflexiona sobre la diferencia entre la inquietud y la necesidad normal de movimiento y exploración a la edad de Jimena. Se menciona la permanente búsqueda de nuevas actividades en la madre para atraer la atención de Jimena, lo que le recuerda la actitud de su madre con ella y sus hermanos. Se refuerza su capacidad para tomar las cosas positivas que recibió de su mamá y tenerlas presente en la relación con Jimena. En esta ocasión, se acuerda incorporar el uso de espacios de juego en el suelo que permitan mayor práctica y autonomía a Jimena.

Sesión 3: Durante la interacción de alimentación se observa un tono emocional bajo, con escasa variaciones y centrado en la tarea. La madre refiere sentirse bien mientras le daba la comida a Jimena, porque se comió todo y luego pudieron jugar.

Trabajo clínico post sesión 3: En las secuencias positivas se observan diálogos en que Juanita pone en palabras los actos y vivencias de la niña: "es un postre para Jimena”, "estás feliz”, “¿está rico?”, y la niña responde. En las secuencias negativas, hay silencios prolongados, juego paralelo, falta de acceso al rostro y conductas controladoras en la madre.

Sesión 4: Durante esta sesión se buscó que Juanita pudiera identificar la relación entre sus emociones y 


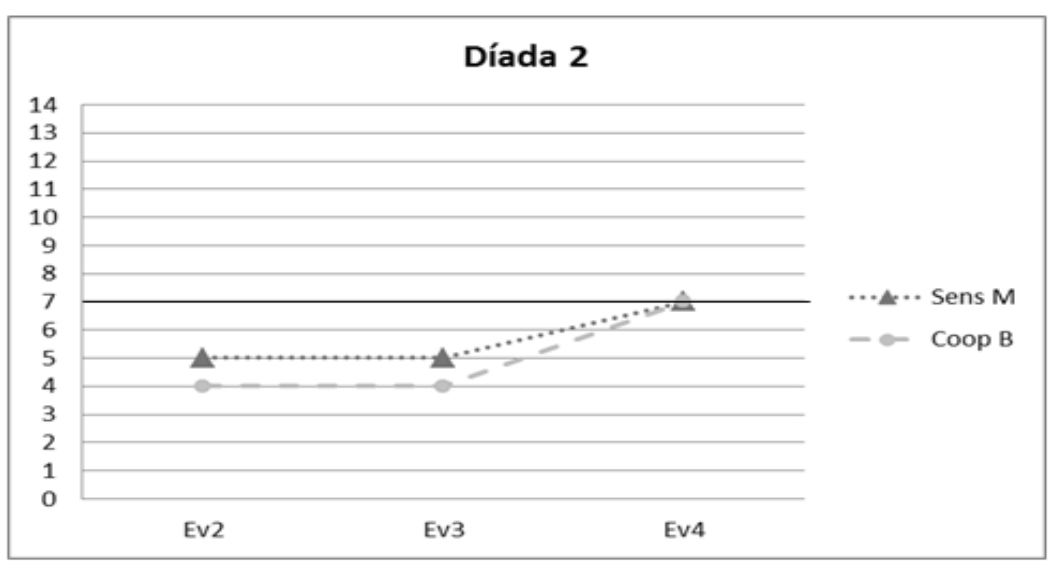

Figura 2. Puntajes obtenidos por la Díada 2 en sensibilidad materna y cooperatividad infantil en las evaluaciones 2, 3 y 4 .

conductas, los afectos positivos de su hija y los momentos de placer compartido. Se observan dificultades en la madre para contener los afectos negativos en la niña y para tolerar diferencias entre ambas en el nivel de activación. Se trabaja en torno a los significados de los distintos estados emocionales y lo que hace o puede hacer para disminuir el malestar.

Cierre: Se revisan los aspectos positivos en las interacciones, destacando la creatividad en la búsqueda de actividades y el poner en palabras lo que cree que le ocurre a la niña. Se recuerdan también los aprendizajes en torno a la necesidad de exploración y práctica en Jimena como parte del desarrollo de mecanismos auto-regulatorios. Juanita logra identificarse con aspectos positivos de su madre, quien murió al tener ella 18 años, pudiendo recordarla desde lo que recibió y no sólo desde la falta. La sensibilidad materna y la cooperatividad de la niña mejoran, ubicándose Juanita en la categoría "adecuada" en la escala de sensibilidad. Los puntajes obtenidos por la Díada 2 en sensibilidad y cooperatividad se presentan en la figura 2 .

\section{Díada 3: Sonia y Juan}

Representaciones y calidad de la interacción: Sonia muestra representaciones negativas en relación a Juan a quien define como "llorón". En la evaluación dos se observa una sensibilidad materna baja que la ubica en la categoría de "riesgo", mejorando en la evaluación tres y ubicándose en la categoría "inepta". $\mathrm{Su}$ interacción inicial con el niño es controladora y con escaso placer, el niño se muestra pasivo e inhibe la expresión de afectos negativos.

\section{Resumen del proceso IG Díada 3}

Sesión 1: Sonia pide ayuda para destetar a Juan, ya que despierta varias veces durante la noche y ella debe amamantarlo en su cama para que se duerma. Esto interfiere en la relación de pareja ya que el padre se va a dormir a la habitación del hijo mayor. Al iniciar la grabación Sonia propone juegos y busca llamar la atención de Juan, quien se adapta a sus propuestas. El juego se focaliza en aspectos cognitivos como encajar, golpear, nombrar, contar y seguir instrucciones. Sonia señala que vio a Juan contento durante el juego y le gustó verlo así, pero que a ella le costó permanecer jugando, "no por compartir con él, sino que ya no sabía qué hacer". Luego expresa que "podría darle más de tiempo para saber con qué le gusta jugar", por lo que se propone como tarea tener un momento diario de juego con Juan.

Trabajo clínico post sesión 1: Las secuencias positivas escogidas muestran cómo el niño acoge las propuestas de la madre y cómo descubre juegos nuevos por sí mismo. Con respecto a los patrones negativos, destaca el control de la madre al momento de proponer juegos y sus dificultades para acomodarse a las necesidades de Juan.

Sesión 2 Retroalimentación, primer video: Se buscó fomentar una adecuada lectura de las señales del niño, identificando emociones y momentos de respuestas ajustadas en la madre. Se muestra un segmento en que Juan descubre un juego y disfruta. Se trabajan también las conductas de exploración y alejamiento, así como contenidos psicoeducativos sobre rutinas de sueño y destete. Finalmente, se muestran segmentos 
que muestran códigos compartidos entre ambos, señalando Sonia al observarlos: "estamos conectados".

Sesión 3: Durante el video de alimentación comparten risas y miradas, Juan se muestra cooperador y busca constantemente participar, tomar la cuchara y acercarse al plato. Sonia limpia la cara de Juan y le explica lo que va a ocurrir, anticipando que a él no le gustará, a raíz de esto se inicia el juego de "está - no está" con la toalla.

Trabajo clínico post sesión 3: Las interacciones positivas muestran momentos en que la madre lee adecuadamente las señales de Juan, lo sigue y respeta sus ritmos. Se destaca la interacción en la limpieza, en que la madre reconoce el estado emocional de su hijo y lo pone en palabras. En cuanto a las interacciones negativas, se observan verbalizaciones y gestos controladores en la madre que impiden la exploración de Juan.

Sesión 4: La madre refiere que las dificultades en el sueño han disminuido y Juan despierta menos en las noches, lo asocia a que ella se mantiene cerca reasegurándolo cuando se despierta. Se muestran segmentos de sintonía, enfatizando la vivencia interna de Juan, en la experiencia de ser reflejado adecuadamente por la madre. Sonia, espontáneamente vincula la experiencia de Juan y su propia experiencia de niña.

Cierre: Se finaliza destacando la capacidad de Sonia para conectarse con su hijo, ser afectuosa y responder a sus necesidades. Se enfatizan también las habilidades de Juan, ligándolas al desempeño materno. Sonia y Juan aumentan la calidad de sus interacciones evolucionando desde la categoría "riesgo" en la escala de sensibilidad a "adecuada", disminuyendo las conductas controladoras de la madre y la pasividad en el niño. La evolución de los puntajes en sensibilidad y cooperatividad se presentan en la figura 3.

A continuación, se presentan los resultados en sintomatología depresiva y cambio terapéutico, considerando para la primera variable los cuatro momentos de evaluación definidos en el programa general y para la segunda las mediciones realizadas al inicio de cada sesión de IG.

\section{Sintomatología depresiva materna y cambio terapéutico}

Los antecedentes en depresión y salud mental de las madres muestran menor gravedad y cronicidad en la díada 1 en comparación con las díadas 2 y 3 . Estas últimas presentaron depresión mayor durante el embarazo $\mathrm{y}$ cuentan con tratamientos previos. Consistentemente, las díadas 2 y 3 obtienen también mayores puntajes en sintomatología depresiva en el BDI-I. Los antecedentes en salud mental de las madres de cada díada se presentan en la tabla 2.

Los mayores puntajes en depresión se presentaron durante el embarazo y disminuyeron hacia el final del

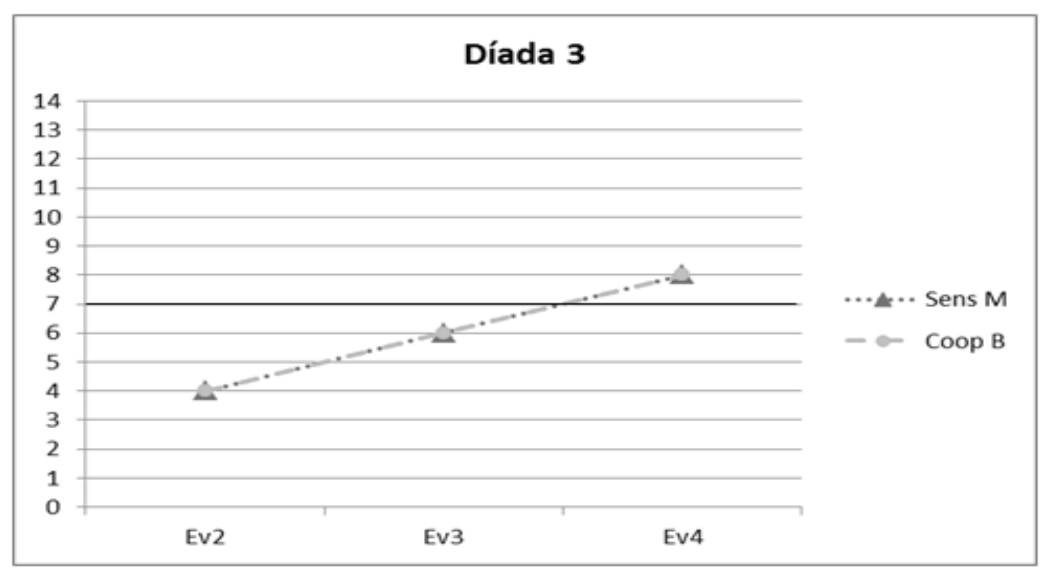

Figura 3. Puntajes obtenidos por la Díada 3 en sensibilidad materna y cooperatividad infantil en las evaluaciones 2,3 y 4 . 
Tabla 2

Antecedentes maternos en depresión y salud mental en las díadas estudiadas.

\begin{tabular}{|c|c|c|c|}
\hline & $\begin{array}{c}\text { Díada } 1 \\
\text { Alejandra - Agustina }\end{array}$ & $\begin{array}{c}\text { Díada } 2 \\
\text { Juanita - Jimena }\end{array}$ & $\begin{array}{c}\text { Díada } 3 \\
\text { Sonia - Juan }\end{array}$ \\
\hline DM embarazo & NO & SI & SI \\
\hline D previa & NO & SI & SI \\
\hline T Psic. Previos & NO & SI por depresión & SI por depresión \\
\hline T Farmac. Previos & SI por sobrepeso & SI por depresión & NO \\
\hline Antec. Familiares & Madre / depresión & Madre / alcoholismo & SA \\
\hline DM fin IG & NO & NO & NO \\
\hline
\end{tabular}

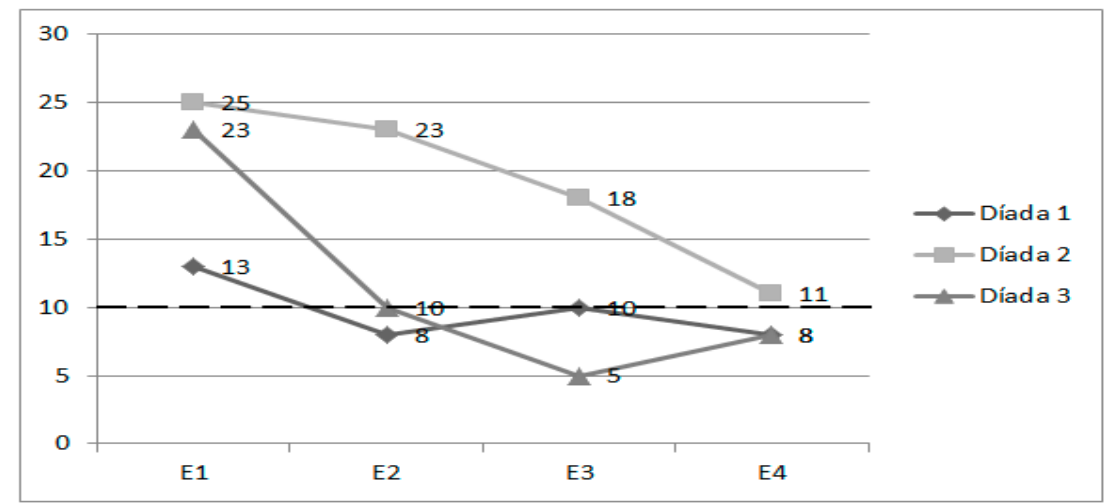

Figura 4. Puntajes obtenidos en sintomatología depresiva materna en las 4 evaluaciones realizadas.

programa, tendencia que se mantiene al finalizar la intervención en IG, ubicándose las tres díadas en la categoría "depresión mínima o no deprimida". Los puntajes obtenidos por las madres en sintomatología depresiva en las 4 evaluaciones se presentan en la figura 4.

Como se esperaba, los niveles de depresión de las madres disminuyen y los niveles de sensibilidad aumentan en el transcurso de las evaluaciones $(\mathrm{E} 1=23$, $\mathrm{E} 2=10, \mathrm{E} 3=10, \mathrm{E} 4=8$ para depresión y E2= 5, E3 $=5, \mathrm{E} 4=7$ para sensibilidad materna), sin embargo, estas diferencias no alcanzan niveles estadísticamente significativos en depresión $\left(X^{2}=6.536, \mathrm{gl}=3, p>0.05\right)$ ni en sensibilidad materna $\left(X^{2}=5.6, \mathrm{gl}=2, p>0.05\right)$. Probablemente la falta de significación estadística de los cambios se explica por el pequeño tamaño muestral.

$\mathrm{Al}$ analizar el cambio terapéutico en las madres durante las cuatro sesiones de IG, se observa una disminución de los puntajes totales en el OQ, que consideran sintomatología ansioso - depresiva, dificultades en las relaciones interpersonales y en el rol social. En la díada
1 la madre reduce su sintomatología en 8 puntos y en las díadas 2 y 3 se observa una reducción de 25 y 17 puntos respectivamente, superando el índice de cambio confiable definido por el instrumento $(\geq 17)$, lo que indica un cambio estadísticamente significativo para estas dos díadas. El detalle de los puntajes obtenidos en cada sesión de IG por las madres en el OQ total se presenta en la figura 5 .

\section{Discusión y Conclusiones}

Como se esperaba, las díadas del estudio presentan una evolución favorable en depresión y sensibilidad materna durante el programa. Los logros en la calidad vincular se incrementan de manera importante en las 3 díadas a partir de la intervención en IG, constituyendo una herramienta que muestra mejores resultados que las intervenciones grupales previas del programa, dirigidas también a esta variable. Se observan cambios en 
Figura 5. Puntajes obtenidos por las madres en el OQ total en cada sesión de IG.

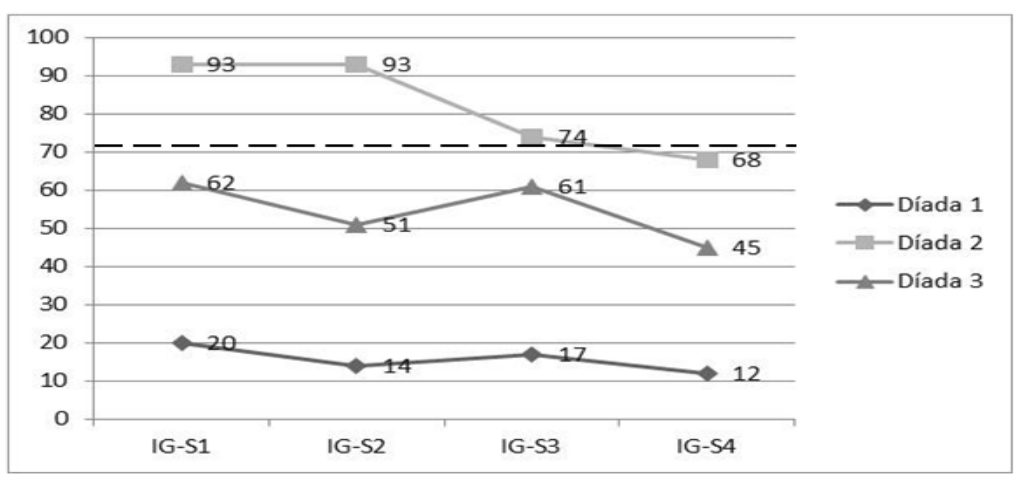

distintos niveles, los que se retroalimentan mutuamente. A nivel individual, las madres reducen su sintomatología depresiva y aumentan su sensibilidad, y los bebés mejoran sus niveles de cooperatividad. A nivel de la relación, mejora la calidad de la interacción y se reduce el riesgo vincular.

Las tres díadas del estudio presentaban estilos inseguros de apego adulto, mostrando las madres de las díadas 1, 2 y 3 estilos evitativo, preocupado y no resuelto respectivamente. Este antecedente alerta sobre las dificultades para establecer relaciones cercanas y seguras, y repercute en la construcción del vínculo con el bebé. Se ha descrito que aquellas personas que presentan un estilo de apego evitativo, como la madre de la díada 1 , manifiestan dificultades para contactarse con los afectos e integrarlos a su experiencia, sobre todo aquellos que son vivenciados negativamente como la tristeza, la rabia y la frustración. Quienes presentan un estilo de apego preocupado, que es el caso de la madre de la díada 2, se muestran abrumadas por temores asociados a preocupaciones por experiencias pasadas. Por último, quienes presentan un estilo de apego temeroso o no resuelto, como la madre de la díada 3 , manifiestan dificultades para integrar afectos y cogniciones a sus experiencias (Main, 1995).

Consistentemente con los planteamientos teóricos expuestos, las tres madres obtienen puntajes iniciales en sensibilidad que indican necesidad de apoyo psicológico, ubicándose en la categoría "inepta" las díadas 1 y 2 y en la categoría "riesgo" la díada 3.

Parte importante de las dificultades de la madre de la díada 1 para reconocer, validar y contener los afectos negativos de su hija podrían explicarse a partir de la dificultad para hacerlo consigo misma, buscando favorecer en la niña el uso de los mecanismos de defensa que ella utiliza frente a las emociones dolorosas. Complementario a esto, los bajos puntajes en sintomatología depresiva podrían explicarse por la falta de contacto con sus afectos más que por la ausencia real de problemas del ánimo, evidenciando una tendencia a utilizar la comida para regular el malestar emocional, confirmándose esto en el sobrepeso de larga data en la madre y recientemente diagnosticado en la niña. En ese sentido, el trabajo realizado con esta díada (1) se focalizó en la integración emocional y el acercamiento de la madre hacia su hija, mediante la identificación de aspectos positivos en ambas que permitieran atenuar las representaciones maternas negativas. Al finalizar la IG, esta díada continúa en el rango que indica necesidad de intervención en relación a la calidad de sus interacciones $(<7)$, lo que señala necesidad de apoyo psicológico de mayor duración para profundizar los cambios y contribuir a la elaboración de la historia vincular de la madre.

La díada 2 presenta el puntaje más alto en sintomatología depresiva materna en todas las mediciones y es la única que obtiene puntajes que indican disfuncionalidad sintomática en el OQ al inicio de la intervención en IG. La dimensión trabajada con esta díada buscó potenciar en la madre el desarrollo de conocimientos ajustados a las necesidades y a la etapa del desarrollo de su hija. De este modo, se buscó corregir las distorsiones en las interpretaciones de las señales de la niña. En este sentido, el reporte que la madre hace del comportamiento de su hija cambia desde una connotación negativa de sus conductas exploratorias hacia una positiva y más ajustada a la experiencia de la niña. Muestra mejorías que la sacan del rango de intervención en relación a la calidad vincular con su hija ( $>7$ en sensibilidad materna) y a la sintomatología general (OQ total $<73$ ). 
El riesgo vincular inicial observado en la díada 3 (4 puntos en sensibilidad materna), podría explicarse a partir de la falta de organización en el estilo de apego materno, asociado a mayor inestabilidad afectiva y comportamental. Al finalizar la IG, la madre de esta díada logra una mejor lectura de las necesidades de su hijo y respuestas adecuadas, dando cuenta de coordinación y sintonía que favorecen la calidad de la interacción. Llama la atención que a pesar de las dificultades iniciales de esta díada, su respuesta a la intervención es mayor que la observada en las díadas 1 y 2 , mejor organizadas en términos de sus estilos de apego. Esto nos lleva a reflexionar sobre las características de las díadas que podrían beneficiarse en mayor medida de intervenciones breves en IG, lo que no necesariamente se asociaría a una menor gravedad en el estilo de apego materno.

$\mathrm{Al}$ analizar de manera global a las tres díadas, es posible destacar que inicialmente todas las madres presentaban dificultades para reconocer y validar las necesidades emocionales en los niños/as y mostraban representaciones negativas sobre sí mismas y/o sus hijos/as. Estas dificultades fueron abordadas destacando los momentos de sintonía, incluyendo la subjetividad de las madres, de los niños/as, y el contexto relacional. En este sentido, la intervención enfatiza los momentos de encuentro placentero entre los miembros de la díada, enriqueciendo el vínculo, la forma en que el bebé experimenta a su madre y cómo ella se experimenta a sí misma (Ogden, 2004).

La mejora en la función reflexiva materna se asoció directamente a un mayor uso de la mentalización en la interacción con los niños/as, lo que incrementó a su vez los momentos de placer y ayudó a las madres a verse y sentirse "buenas madres". Asimismo, el reconocimiento de momentos positivos les permitió abordar sus dificultades de un modo constructivo, logrando identificar "buenas prácticas" para influir en el estado emocional de sus hijos/as.

Aún cuando las diferencias encontradas entre los niveles de depresión y sensibilidad a lo largo de la intervención no son estadísticamente significativos, muestran una tendencia positiva al cambio, en tanto que los niveles de depresión disminuyen y los de sensibilidad aumentan, cambio que es mayor entre las evaluaciones 3 y 4 , es decir, antes y después de la intervención con videofeedback. Complementario a esto, los cambios observados en sintomatología clínica evaluada con el OQ, muestran una mejoría estadísticamente significativa en las díadas 2 y 3 . Estos resultados denotan la necesidad de robustecer los análisis con una muestra de mayor tamaño, de manera de poder definir con mayor rigor el efecto de la modalidad de intervención desarrollada.

Los resultados muestran que a partir de la construcción de un espacio protegido de observación y reflexión es posible conocer las representaciones previas de las madres y contrastarlas con las que emergen a partir de los distintos segmentos observados en los videos, contribuyendo la experiencia de la terapeuta con la madre y la de la co-terapeuta con el bebé durante las sesiones a enriquecer la comprensión del caso.

Resulta importante destacar que la intervención en IG puede ser utilizada tempranamente en el desarrollo infantil, a un bajo costo y beneficiando al niño/a, a la madre y el vínculo. A pesar de esto, los resultados deben ser tomados con cautela ya que el estudio representa una intervención piloto que requiere ser probada en muestras de mayor tamaño, con un grupo de control o de comparación.

\section{Referencias}

Allen, J., \& Fonagy, P. (2002). The Development of mentalizing and its role in psychopathology and psychotherapy (Reporte técnico No. 02-0048). Houston: Menninger Clinic.

Allen, J., Fonagy, P. \& Bateman, (2008). Mentalizing in Clinical Practice. London: American Psychiatric Publishing, Inc.

Alvarado, R., Rojas, M., Monardes, J., Perucca, E., Neves, E., Olea, E. \& Vera, A. (2000). Cuadros depresivos en el postparto en una cohorte de embarazadas: Construcción de un modelo causal. Revista Chilena de Neuro-Psiquiatría, 38(2), 84-93. doi:10.4067/ S0717-92272000000200003

Beck, A., Ward, C., Mendelson, M., Mock, J. \& Erbaugh, J. (1961). An inventory for measuring depression. Archives of General Psychiatry, 4(6), 561-571. doi:10.1001/archpsyc.1961.01710120031004

Biedermann, K., Martínez, V., Olhaberry, M. \& Cruzat, C. (2009). Aportes de la Teoría del Apego al tratamiento de los trastornos de Alimentación: Implicancias Clínicas. Revista Argentina de Clínica Psicológica, 18, 217-226. Recuperado de http://www.cienciared. com.ar/ra/doc.php?n=1142

Cohn, J.F., Matias, R., Tronick, E. Z., Connell, D. \& Lyons- Ruth, K (1986). Face-to-face interactions of depressed mothers and their infants. New Direction for Child and Adolescent Development, 34, 31-45. doi:10.1002/cd.23219863405

Cornish, A., McMahon, C., Ungerer, J., Barnett, B., Kowalenko, N. \& Tennet, C. (2006). Maternal depression, the experience of parenting in the second postnatal year. Journal of Reproductive and Infant Psychology, 24(2), 121-132. doi:10.1080/02646830600644021

Crittenden, P. (2006). CARE-Index para Infantes (Nacimiento-15 meses) Manual de Codificación. Family Relations Institute, Miami USA.

DiCarlo, C., Onwujuba, Ch. \& Baumgartner, J. (2013). Infant Comunicative Behavior and Maternal Responsiveness. New York: Springer, Science and Business Media.

Earls, M. (2010). Incorporating Recognition and Management of Perinatal and Postpartum Depression into Pediatric Practice. Pediatrics, 126(5), 1032-1039. doi:10.1542/peds.2010-2348

Evans, G., Vicuña, M. \& Marín, R. (2003). Depresión posparto: Realidad en el sistema público de atención de salud. Servicio de ob- 
stetricia y ginecología. Revista Chilena Obstetricia Ginecológica, 68(6), 491-494. doi:10.4067/S0717-75262003000600006

Field, T., Diego, M. \& Hernandez-Reif, M. (2006). Prenatal depression effects on the fetus and newborn: a review. Infant Behavior and Development, 29(3), 445-455. doi:10.1016/j. infbeh.2006.03.003

Field, T. (2011). Prenatal depression effects on development. Infant Behavior and Development, 34(1), 1-14. doi:10.1016/j. infbeh.2010.09.008

Fonagy, P. (1997). Attachment and theory of mind: Overlapping constructs?. Association for Child Psychology and Psychiatry Occasional papers, 14, 31-40. Recuperado de http://discovery. ucl.ac.uk/id/eprint/134186

Fonagy, P., Bateman, A. \& Bateman, A. (2011). The widening scope of mentalizing: a discussion. Psychology ans Psychotherapy: Theory, Research and Practice, 84(1), 98-110. doi:10.1111/j.20448341.2010.02005.x

Gómez, E. \& Muñoz, M. (2013). Manual para el modelo de videofeedback. oportunidades para el desarrollo de interacciones sensibles, eficaces y afectivas. Manuscrito inédito.

Halbreich, U. \& Karkun, S. (2006). Cross-cultural and social diversity of prevalence of postpartum depression and depressive symptoms. Journal of Affective Disorders, 91(2-3), 97-111. doi:10.1016/j. jad.2005.12.051

Juffer, F., Bakermans-Kranenburg, M. J. \& van Ijzendoorn M. H. (2008). Promoting positive parenting. An attachment-Based Intervention. New York: Psychology Press.

Lambert, M. J., Hansen, N. B., Umpress, V., Lunnen, K. \& Okiishi, J. (1996). Administration and Scoring Manual for the OQ-45.2. American Professional Credentialing Services LLC. USA.

Main, M. (1995). Recent studies in attachment: Overview, with selected implications for clinical work. En S. Goldberg, R. Muir, \& J. Kerr (Eds.), Attachment theory: Social, developmental, and clinical perspectives (pp. 407-474). New York: Analytic Press.

McDonough, S. (1993). Interaction guidance: Understanding and treating early infant-caregiver relationship disturbances. En C. H. Zeanah (Ed.), Handbook of Infant Mental Health (pp. 414-426). New York: The Guilford Press.

McDonough, S. C. (2004). Interaction Guidance: Promoting and nurturing the caregiving relationship. En A. Sameroff, S. C. McDonough, \& K. L. Rosenblum, (Eds.) Treating parent-infant relationship problems: Strategies for intervention (pp. 79-96). New York: Guilford Press.

Ministerio de Salud de Chile (2013). Guía clínica AUGE depresión en personas de 15 años y más. Serie Guías Clínicas Minsal, 2013. Recuperado de http://web.minsal.cl/portal/url/item/7222754637c 08646e04001011f014e64.pdf

Minkovitz, C., Strobino, D., Scharfstein, D., Hou, W., Miller, T., Mistry, C. \& Swartz, K. (2005). Maternal Depressive Symptoms and Children's Receipt of Health Care in the First 3 Years of Life. Pediatrics, 115(2), 306-314, doi:10.1542/peds.2004-0341

Muzik, M. \& Borovska, S. (2010). Perinatal depression: implications for child mental health. Mental Health in Family Medicine, 7(4), 239-247. Recuperado de http://www.mhfmjournal.com/ mental-health/perinatal-depression-implications-for-child-mentalhealth.pdf
O'Connor, T. \& Zeanah, C. (2003). Attachment disorders: Assessment strategies and treatment approaches. Attachment \& Human Development, 5(3), 223-244. doi:10.1080/1461673031000159 3974

Ogden, T. (2004). On Holding and Containing, being in dreaming. International Journal of Psychoanalysis, 85(6), 1349-1364. doi:10.1516/T41H-DGUX-9JY4-GQC7

Olhaberry, M., Escobar, M., San Cristóbal, P., Santelices, M., Farkas, Ch., Rojas, G. \& Martínez, V. (2013). Intervenciones psicológicas perinatales en depresión materna y vínculo madre-bebé: una revisión sistemática. Terapia Psicológica, 31(2), 249-261. doi:10.4067/S0718-48082013000200011

Olhaberry, M. \& Santelices M. P. (2013). Presencia del padre y calidad de la interacción madre-hijo(a): un estudio comparativo en familias chilenas nucleares y monoparentales. Universitas Psychologica, 12(3), 833-843. doi:10.11144/Javeriana.UPSY123.ppci

Pearson, R., Melotti, R., Heron, J., Joinson, J., Stein, A., Ramchandani, P. \& Evans, A. (2012). Disruption to the development of maternal responsiveness? The impact of prenatal depression on mother-infant interactions. Infant, Behavior \& Development, 35(4), 613-626. doi:10.1016/j.infbeh.2012.07.020

Rojas, G., Fritsch, R., Solis, J., Jadresic, E., Castillo, C., González, M., ... \& Araya, R. (2007). Treatment of postnatal depression in low-income mothers in primary-care clinics in Santiago, Chile: a randomized controlled trial. Lancet, 370(9599), 1629-1637. doi:10.1016/S0140-6736(07)61685-7

Santelices, M. P., Olhaberry, M., Araneda, M. E., Tapia, C. \& PérezSalas, C. P. (2007). Estudio de la evolución de las representaciones y apego materno. Revista Argentina de Clínica Psicológica, 16(3), 219-229. Recuperado de http://www.redalyc.org/articulo. oa? id $=281921790003$

Suárez, N., Muñoz, M., Gómez, E. \& Santelices, M. (2009). Terapia de Interacción Guiada: Una Nueva Modalidad de Intervención con Familias Multiproblemáticas y en Riesgo Social. Terapia Psicológica, 27(2), 203-213. doi:10.4067/S071848082009000200006

Vik, K. \& Braten, S. (2009). Video interaction guidance inviting transcendence of postpartum depressed mothers' self-centered state and holding behavior. Infant Mental Health Journal, 30(3), 287-300. doi:10.1002/imhj.20215

Vik, K. \& Hafting, M. (2006). Video interaction guidance offered to mothers with postnatal depression: experiences from a pilot study. Nordic Journal of Psychiatry, 60(3), 234-238. doi:10.1080/08039480600636593

Von Bergen, A. \& De la Parra, G. (2002). OQ-45.2, Cuestionario para evaluación de resultados y evolución en psicoterapia: Adaptación, validación e indicaciones para su aplicación e interpretación. Terapia Psicológica, Sociedad Chilena de Psicología Clínica, 20(2), 161-176. Recuperado de http://goo.gl/UjTVzg

Wei, M., Russell, D. W., Mallinckrodt, B. \& Vogel, D. L. (2007). The experiences in Close Relationship Scale (ECR)-Short Form: Reliability, validity, and factor structure. Journal of Personality Assessment, 88(2), 187-204. doi:10.1080/00223890701268041 